\title{
A NOTE ON TWO CONGRUENCES ON A GROUPOID
}

\author{
K. NIRMALA KUMARI AMMA
}

ABSTRACT. Let $S$ be a groupoid and $\theta_{p}, \theta_{m}$ the congruences on $S$ defined as follows: $x \theta_{p} y\left(x \theta_{m} y\right)$ iff every prime (minimal prime) ideal of $S$ containing $x$ contains $y$ and vice versa. It is proved that $\theta_{p}$ is the smallest congruence on $S$ for which the quotient is a semilattice. It is also shown that $S / \theta_{m}$ is a disjunction semilattice if $S$ has $\mathbf{0}$ and is a Boolean algebra if $S$ is intraregular and closed for pseudocomplements. Some connections between the ideals of $S$ and those of the quotients are established. Congruences similar to $\theta_{p}$ and $\theta_{m}$ are defined on a lattice using lattice-ideals; quotients under these are distributive lattices.

Introduction. This paper consists of four sections. $\$ 1$ is devoted to a summary of some results on ideals and pseudocomplements which are used in subsequent sections. In $\$ 2$ we define two congruences on a groupoid $S$ in terms of prime ideals. The quotients under these are semilattices. Relations between the ideals of $S$ and those of the quotients are investigated. Theorems 3 and 6 are the main theorems in this direction. $\$ 3$ deals with the additional properties of the congruences. Two congruences on a lattice are given in $\$ 4$. Some connected results for commutative semigroups and lattices have been proved by Kist [3], Varlet [5] and Venkatanarasimhan [6]. For standard concepts and notations used in this paper, the reader may refer to Birkhoff [1] and Clifford and Preston [2].

1. Preliminaries. By an ideal we mean an ideal in the sense of [2] and by a lattice-ideal we mean an ideal in the sense of [1]. Let $S$ be a groupoid. A nonempty subset $A$ of $S$ is called a filter if $a, b \in A \Leftrightarrow a b \in A$. The smallest filter containing an element $a$ is called the principal filter generated by $a$ and is denoted by $K(a)$. The principal ideal generated by $a$ is denoted by $J(a)$. An ideal $|A|$ of $S$ is said to be prime if $a b \in A \Rightarrow a \in A$ or $b \in A$. $S$ is said to be intraregular if $J(a b)=J(a) \cap J(b)$ for any two elements $a, b$ of $S$. If $S$ has 0 and $a \in S$, by the pseudocomplement of $a$ we mean an element $a^{*}$ of $S$ such that $a a^{*}=0=a^{*} a$ and $a b=0=b a \Rightarrow b a^{*}=b=a^{*} b$. We shall denote lattice-join and lattice-meet by the symbols $\vee$ and $\wedge$ respectively.

We need the following lemmas in the sequel.

LEMMA I [4]. A nonempty proper subset $A$ of a groupoid $S$ is a prime (minimal

Received by the editors July 12, 1976 and, in revised form, January 19, 1977.

AMS (MOS) subject classifications (1970). Primary 20L99, 06A20; Secondary 06A35, 06A40.

Key words and phrases. Groupoid, intraregular, prime ideal, filter, congruence, semilattice, disjunction property, pseudocomplement, Boolean algebra. 
prime) ideal of $S$ if and only if $S-A$ is a filter (maximal filter) of $S$.

LEMMA II [4]. Any prime ideal (proper filter) of a groupoid with 0 contains (is contained in) a minimal prime ideal (maximal filter).

LEMMA III [4]. A groupoid $S$ is intraregular if and only if every ideal of $S$ is an intersection of prime ideals.

LEMMA IV [4]. In an intraregular groupoid $S$ with 0 , a prime ideal is minimal prime if and only if it contains precisely one $J(x), J(x)^{*}$ for every $x \in S$. In an intraregular groupoid $S$ closed for pseudocomplements, a prime ideal is minimal prime if and only if it contains precisely one of $x, x^{*}$ for every $x \in S$.

LEMMA V [4]. In an intraregular groupoid with 0 , the pseudocomplement of an ideal is the intersection of all the minimal prime ideals not containing it.

LEMMA VI [7]. A semilattice $S$ with 0 is a disjunction semilattice if and only if distinct principal ideals of $S$ have distinct pseudocomplements.

LEMMA VII [7]. A disjunction semilattice closed for pseudocomplements is a Boolean algebra.

LeMma VIII (cf. [2, p. 12, Example 1]). Let $f$ be a homomorphism of a groupoid $S$ onto a groupoid $T$. Then:

(i) If $A$ is an ideal of $S, f(A)$ is an ideal of $T$. If $B$ is an ideal of $T, f^{-1}(B)$ is an ideal of $S$.

(ii) If $A$ is a prime ideal (filter) of $T, f^{-1}(A)$ is a prime ideal (filter) of $S$.

(iii) $f(J(x))=J(f(x))$ for all $x \in S$.

Proofs are not given for the results on filters since they may be inferred from the ones for prime ideals using Lemma I.

2. Two congruences on a groupoid. Throughout this section, $S$ will denote a groupoid.

For $x, y \in S$ define $x \theta_{p} y\left(x \theta_{m} y\right)$ to mean that every prime (minimal prime) ideal of $S$ containing $x$ contains $y$ and vice versa. It is easily seen that $\theta_{p}$ and $\theta_{m}$ are congruences on $S$ and that $S / \theta_{p}$ and $S / \theta_{m}$ are semilattices. We shall denote the natural homomorphism of $S$ onto $S / \theta_{p}\left(S / \theta_{m}\right)$ by $\theta_{p}^{*}\left(\theta_{m}^{*}\right)$.

LEMMA 1. Let $A$ be a prime ideal or a filter (minimal prime ideal or a maximal filter) of $S$. Then $\theta_{p}^{*-1} \theta_{p}^{*}(A)=A\left(\theta_{m}^{*-1} \theta_{m}^{*}(A)=A\right)$.

Proof. Clearly the prime (minimal prime) ideals of $S$ are unions of $\theta_{p}$ $\left(\theta_{m}\right)$-classes. Hence the lemma follows.

THEOREM 1. If $A$ is any prime ideal (filter) of $S$, then $\theta_{p}^{*}(A)$ is a prime ideal (filter) of $S / \theta_{p}$. If $A$ is a minimal prime ideal (maximal filter) of $S$, then $\theta_{p}^{*}(A)$ is a minimal prime ideal (maximal filter) of $S / \theta_{p}$ and $\theta_{m}^{*}(A)$ is a minimal prime ideal (maximal filter) of $S / \theta_{m}$.

Proof. Let $A$ be a prime ideal of $S$. Clearly, $x, y \in S, \theta_{p}^{*}(x) \theta_{p}^{*}(y) \in \theta_{p}^{*}(A)$ 
$\Rightarrow x y \in A \Rightarrow x \in A$ or $y \in A \Rightarrow \theta_{p}^{*}(x) \in \theta_{p}^{*}(A)$ or $\theta_{p}^{*}(y) \in \theta_{p}^{*}(A)$. Hence $\theta_{p}^{*}(A)$ is a prime ideal.

If $A$ is a minimal prime ideal and $B$ is any prime ideal of $S / \theta_{p}$ such that $B \subseteq \theta_{p}^{*}(A)$, then $\theta_{p}^{*-1}(B) \subseteq A$ by Lemma 1 . Also $\theta_{p}^{*-1}(B)$ is prime by Lemma VIII. Hence $\theta_{p}^{*-1}(B)=A$ and so $\theta_{p}^{*}(A)=B$. Thus $\theta_{p}^{*}(A)$ is minimal prime. The last part is proved on similar lines.

TheOREM 2. If $A$ is a minimal prime ideal (maximal filter) of $S / \theta_{p}$, then $\theta_{p}^{*-1}(A)$ is a minimal prime ideal (maximal filter) of $S$. If $S$ has 0 and $A$ is a minimal prime ideal (maximal filter) of $S / \theta_{m}$, then $\theta_{m}^{*-1}(A)$ is a minimal prime ideal (maximal filter) of $S$.

Proof. Let $A$ be a minimal prime ideal of $S / \theta_{p}$. By Lemma VIII, $\theta_{p}^{*-1}(A)$ is a prime ideal of $S$. Let $B$ be any prime ideal of $S$ such that $\theta_{p}^{*-1}(A) \supseteq B$. Then $A \supseteq \theta_{p}^{*}(B)$. By Theorem 1 and Lemma 1 , it follows that $\theta_{p}^{*-1}(A)=B$. Hence $\theta_{p}^{*-1}(A)$ is a minimal prime ideal of $S$.

Suppose $S$ has 0 and $A$ is a minimal prime ideal of $S / \theta_{m}$. By Lemma VIII, $\theta_{m}^{*-1}(A)$ is prime. By Lemma II, $\theta_{m}^{*-1}(A) \supseteq B$ for some minimal prime ideal $B$ of $S$. As in the first part, $\theta_{m}^{*-1}(A)=B$. Thus, $\theta_{m}^{*-1}(A)$ is minimal prime.

As a consequence of Theorems 1 and 2 and Lemma VIII, we have the following.

THEOREM 3. There is a bijection between the set of prime ideals (filters) of $S$ and the set of prime ideals (filters) of $S / \theta_{p}$. Under this bijection, the minimal prime ideals (maximal filters) of $S$ correspond to the minimal prime ideals (maximal filters) of $S / \theta_{p}$. If $S$ has 0 , there is a bijection between the set of minimal prime ideals (maximal filters) of $S$ and the set of minimal prime ideals (maximal filters) of $S / \theta_{m}$.

THEOREM 4. If $\left\{A_{i}: i \in I\right\}$ is a family of prime ideals or filters (minimal prime ideals or maximal filters) of $S$, then

$$
\theta_{p}^{*}\left(\bigcap_{i \in I} A_{i}\right)=\bigcap_{i \in I} \theta_{p}^{*}\left(A_{i}\right)\left(\theta_{m}^{*}\left(\bigcap_{i \in I} A_{i}\right)=\bigcap_{i \in I} \theta_{m}^{*}\left(A_{i}\right)\right) .
$$

Proof. Let $x \in \cap_{i \in I} \theta_{p}^{*}\left(A_{i}\right)$. Then for each $i \in I$, there exists $x_{i} \in A_{i}$ such that $x=\theta_{p}^{*}\left(x_{i}\right)$. It follows that $x \in \theta_{p}^{*}\left(\cap_{i \in I} A_{i}\right)$. Thus $\bigcap_{i \in I} \theta_{p}^{*}\left(A_{i}\right) \subseteq$ $\theta_{p}^{*}\left(\cap_{i \in I} A_{i}\right)$. The reverse inclusion is obvious.

The second part is proved on similar lines.

As a consequence of Lemmas 1 and III and Theorem 4, we have the following.

Corollary 1. If $S$ is intraregular and $A$ is an ideal of $S$, then $\theta_{p}^{*-1} \theta_{p}^{*}(A)=$ $A$.

Corollary 2. If $S$ is intraregular and $\left\{A_{i}: i \in I\right\}$ is a family of ideals of $S$, then $\theta_{p}^{*}\left(\cap_{i \in I} A_{i}\right)=\bigcap_{i \in I} \theta_{p}^{*}\left(A_{i}\right)$.

The following theorem is easily proved. 
THEOREM 5. For each $x \in S, \theta_{p}^{*}(K(x))=K\left(\theta_{p}^{*}(x)\right)$. If $\left\{A_{i}: i \in I\right\}$ is a family of filters of $S, \theta_{p}^{*}\left(\bigvee_{i \in I} A_{i}\right)=\bigvee_{i \in I} \theta_{p}^{*}\left(A_{i}\right)$. If $\left\{B_{i}: i \in I\right\}$ is a family of filters of $S / \theta_{p}, \theta_{p}^{*-1}\left(\bigvee_{i \in I} B_{i}\right)=\bigvee_{i \in I} \theta_{p}^{*-1}\left(B_{i}\right)$.

As an immediate consequence of Theorems 3, 4 and 5, Corollaries 1 and 2 and Lemma VIII, we have the following.

THEOREM 6. If $S$ has $1, \theta_{p}^{*}$ induces an isomorphism between the lattice of filters of $S$ and the lattice of filters of $S / \theta_{p}$ which preserves unrestricted joins, unrestricted meets, the property of being a principal filter and the property of being a maximal filter in both directions. If $S$ is an intraregular groupoid with 0 , $\theta_{p}^{*}$ induces an isomorphism between the lattice of ideals of $S$ and the lattice of ideals of $S / \theta_{p}$ which preserves unrestricted joins, unrestricted meets, the property of being a principal ideal, the property of being a prime ideal and the property of being a minimal prime ideal in both directions.

THEOREM 7. If $S$ is an intraregular groupoid with 0 and $A$ is any ideal of $S$, then $\theta_{m}^{*}\left(A^{*}\right)=\theta_{m}^{*}(A)^{*}$.

Proof. Let $\left\{A_{i}: i \in I\right\}$ be the family of minimal prime ideals of $S$ not containing $A$. Then the ideals $\theta_{m}^{*}\left(A_{i}\right)$ are precisely the minimal prime ideals of $S / \theta_{m}$ not containing $\theta_{m}^{*}(A)$. By Lemma $V$ and Theorem 4 it follows that $\theta_{m}^{*}\left(A^{*}\right)=\theta_{m}^{*}(A)^{*}$.

3. Additional properties of $\theta_{p}$ and $\theta_{m}$. As in $\S 2, S$ will denote a groupoid throughout this section.

THEOREM 8. Let $\theta$ be any congruence on $S$. Then $S / \theta$ is a semilattice if and only if $\theta_{p} \subseteq \theta$.

Proof. The 'If part' is obvious. Suppose $S / \theta$ is a semilattice and let $x \theta_{p} y$. Let $\theta^{*}$ denote the natural homomorphism of $S$ onto $S / \theta$. It is easily seen that $J\left(\theta^{*}(x)\right)=J\left(\theta^{*}(y)\right)$. Hence $\theta^{*}(x)=\theta^{*}(y)$. Thus $\theta_{p} \subseteq \theta$.

TheOREM 9. For any two distinct elements of $S / \theta_{m}$, there exists a minimal prime ideal of $S / \theta_{m}$ containing exactly one of them.

Proof. Let $\theta_{m}^{*}(x), \theta_{m}^{*}(y)$ be any two distinct elements of $S / \theta_{m}$ so that $x, y \in S$. Then there exists a minimal prime ideal $A$ of $S$ containing exactly one of $x, y$, say $x$. Clearly, $\theta_{m}^{*}(A)$ is a minimal prime ideal of $S / \theta_{m}$ such that $\theta_{m}^{*}(x) \in \theta_{m}^{*}(A)$ and $\theta_{m}^{*}(y) \notin \theta_{m}^{*}(A)$.

As a consequence of Theorem 9 and Lemmas IV and VI, we have the following.

THEOREM 10. If $S$ is an intraregular groupoid with 0 , then $S / \theta_{m}$ is a disjunction semilattice.

THEOREM 11. Let $S$ be an intraregular groupoid closed for pseudocomplements. Then $x \theta_{m} y \Leftrightarrow x^{*}=y^{*}$ and $S / \theta_{m}$ is a Boolean algebra.

Proof. By Lemma IV it follows that $x \theta_{m} y \Leftrightarrow x^{*}=y^{*}$. It is easily seen that 
$\theta_{m}^{*}\left(x^{*}\right)$ is the pseudocomplement of $\theta_{m}^{*}(x)$ for each $x \in S$. By Theorem 10 and Lemma VII it follows that $S / \theta_{m}$ is a Boolean algebra.

4. Two congruences on a lattice. Throughout this section $L$ will denote a lattice.

For $x, y \in L$ define $x \phi_{p} y\left(x \phi_{m} y\right)$ to mean that every prime lattice-ideal (minimal prime lattice-ideal) containing $x$ contains $y$ and vice versa. Then it is easily seen that $\phi_{p}$ and $\phi_{m}$ are congruences on $L$ and that $L / \phi_{p}$ and $L / \phi_{m}$ are distributive lattices.

Analogues of many of the results in $\S \S 2$ and 3 hold for $\phi_{p}$ and $\phi_{m}$. For example, we have the following.

THEOREM 12. Let $\theta$ be any congruence on $L$. Then $L / \theta$ is a distributive lattice if and only if $\phi_{p} \subseteq \theta$.

Acknowledgement. The author is indebted to Dr. P. V. Venkatanarasimhan for his guidance and help in preparing this note. She is thankful to the referee for some suggestions.

\section{REFERENCES}

1. G. Birkhoff, Lattice theory, Amer. Math. Soc. Colloq. Publ., vol. 25, Amer. Math. Soc., Providence, R.I., 1948.

2. A. H. Clifford and G. B. Preston, The algebraic theory of semigroups, Vol. I, Math. Surveys, no. 7, Amer. Math. Soc., Providence, R.I., 1961.

3. J. E. Kist, Minimal prime ideals in commutative semigroups, Proc. London Math. Soc. 13 (1963), 31-50.

4. K. Nirmala Kumari Amma, Pseudocomplements in groupoids (to appear).

5. J. Varlet, Congruences dans les treillis pseudocomplémentes, Bull. Soc. Roy Sci. Liège 9 (1963), 623-635.

6. P. V. Venkatanarasimhan, A note on modular lattices, J. Indian Math. Soc. 30 (1966), 55-59.

7. , Semi-ideals in semilattices, Colloq. Math. 30 (1974), 203-212.

Department of Mathematics, University of Kerala, Kariavattom, Trivandrum, India 